\title{
Shear Bond Strength of Polymeric to Dentin
}

\author{
Linfu Han, Lijun Liu* , Huanhuan Zhang \\ Email address: \\ 513255873@qq.com (Linfu Han),761079080@qq.com (Lijun Liu) \\ ${ }^{*}$ Corresponding author
}

Department of Stomatology, The Fourth Affiliated Hospital of Inner Mongolia Medical University, Baotou, China

To cite this article:

Linfu Han, Lijun Liu, Huanhuan Zhang. Shear Bond Strength of Polymeric to Dentin. International Journal of Clinical Oral and Maxillofacial Surgery. Vol. 6, No. 2, 2020, pp. 44-48. doi: 10.11648/j.ijcoms.20200602.15

Received: March 9, 2020; Accepted: March 23, 2020; Published: September 25, 2020

\begin{abstract}
The purpose of this study was to evaluate the dentin bond strength of restorations made of different polymeric materials with Single Bond Universal Adhensive and etch-and-bond resin cement. Ceromer (ceramage, Shofu), Polymethylmethacrylate (PMMA DISK, Yamahachi), resin nanoceramic (Lava Ultimate, 3M ESPE), and polymer-infiltrated-ceramic-network (Vita Enamic, Vita Zahnfabrik) specimens (n=15/group) were fabricated respectively. Dentin specimens were prepared from extracted third molars stored in distilled water in a refrigerator $\left(4^{\circ} \mathrm{C}\right)$. Single Bond Universal Adhensive was applied to the prepared tooth and light cured. Then, specimens were cemented using 3M ESPE RelyX ${ }^{\mathrm{TM}}$ Uitimate Cliker ${ }^{\mathrm{TM}}$ adhesive resin cement. All cemented specimens were stored in distilled water for $24 \mathrm{~h}$ and subjected to shear forces by a universal testing machine. Vita Enamic was found to show the highest shear bond strength values. The shear bond strength of Lava Ultimate was weaker than that of Vita Enamic. But there was no statistical difference between Vita Enamic and Lava Ultimate. Both of them showed significantly higher shear bond strength than the Ceramage and PMMA groups. The lowest values were obtained for PMMA and there was a significant difference between the PMMA and Ceramage groups. The bond strength of the polymeric materials is material dependent. So doctors can use them for patients with different intent.
\end{abstract}

Keywords: Ceramage, Polymethylmethacrylate, Resin Nanoceramic, Polymer-infiltrated-ceramic-network, Shear Bond Strength

\section{Introduction}

In recent decades, metal-free, tooth-colored and high-performance restorations, such as ceramics or polymeric materials, have been used to replace missing tooth structures to rehabilitate the esthetics and functions of defected teeth. The advantage of ceramic restorations is that ceramics have superior esthetic appearance, good biocompatibility, durability, color stability [1], and can mimic the structural characteristics of natural teeth [2]. However, many studies have shown that ceramics are strong, rigid, brittle materials, with high susceptibility to fracture [3], and can induce damage to the natural surface of the tooth [4]. Recently, polymeric materials have been introduced for dental restorations [5], and with continuous evolution of polymeric materials have led to materials that have esthetic appearance, higher abrasion resistance and inferior discoloration [6], as well as less abrasive effect on the antagonist enamel [7-9].

It has been reported that one of the key advantages of polymeric restorations is the low abrasiveness of the enamel antagonists in comparison with ceramics [10-12]. Another advantage is the low elastic modulus, which allows better absorption of functional stresses by deformation [13]. Some studies have pointed out that ceramic restorations require certain thickness to acquire adequate fracture resistance, but for polymer restorations, even ultrathin polymeric materials can show higher fracture resistance than ceramics $[14,15]$. Ender et al. [16] reported that, based on the findings of marginal adaptation, fracture load and macroscopic fracture mode, polymeric materials may be applicable as long-term restorations in some cases. In view of these advantages, polymeric materials have been considered as economic alternatives for ceramics with faster processing, higher performance, and lower costs $[17,18]$.

Despite the expanded application of polymeric materials, the clinical longevity of these restorations is still regulated by the strong and durable bonding to both materials and tooth with resin cement. Bonding properties of these polymeric materials still needs to be investigated, especially Ceramage 
and PMMA, as there is a lack of information on the shear bond strength of polymeric materials and dentin in the literatures. The objective of this study is to compare the shear bond strength between different polymeric materials and dentin.

\section{Methods}

\subsection{Preparation of Dentin Specimens}

60 human third permanent molars from 16-year-old to 40-year-old individuals were extracted. The criteria for tooth selection included intact enamel with no cracks caused by extraction, the absence of caries, and an adequate dimension of the crown [17]. Before extraction, patients have been informed about the use of the teeth for research purposes, and verbal consent have been obtained. The teeth were thoroughly washed in running water and all blood and adherent tissue were removed by the clinician with periodontal curettes and stored in distilled water in a refrigerator $\left(4^{\circ} \mathrm{C}\right)$ according to the ISO 11405:2015 (Dentistry-Testing of adhesion to tooth structure) until needed. The occlusal surface of each tooth was wet ground in an automatic grinding machine with rotating abrasive discs to remove enamel and to expose a $5 \mathrm{~mm}$ area of dentin, and the roots of the teeth were mounted in a holder with self-curing acrylic resin. In order to ensure correct alignment in the test fixtures, the ground surface of each tooth was parallel to the shearing force. The exposed dentin surfaces were prepared by planning against silicon carbide abrasive paper with a grit size of P120 and P400 by a two-step sequential planning process under running water to obtain an even and uniform dentin surface and reduce any micromechanical interlocking that could affect the real bonding influence of the tested adhesive cements (ISO 29022:2013 Dentistry-Adhesion-Notched-edge shear bond strength test).
Before cementation, to remove any foreign matter, the dentin surface was cleaned for $1 \mathrm{~min}$ with a cotton pellet impregnated with alcohol and ultrasonically cleaned in distilled water for $5 \mathrm{~min}$. The mounted tooth was then placed in water at room temperature and use for the bonding procedure within $4 \mathrm{~h}$. The surface was then rinsed and dried with an oil-free air stream before cementation. The teeth were randomly divided into 4 groups. One operator carried out all procedures to maximize standardization.

\subsection{Preparation of Polymeric Specimens}

Groups 1:15 Ceramage specimens were fabricated by placing the Ceramage inside a mold made of a Teflon material with an inner diameter of $4 \mathrm{~mm}$ and a height of $2 \mathrm{~mm}$, and then light-polymerised with Solidilight (Shofu Inc., Kyoto, Japan) according to the manufacturer's recommendations. A glass microscope slide was used to compress the last increment to obtain a flat surface. After polymerization, specimens were removed from the mold.

Groups 2: PMMA specimens were milled from PMMA DISK (Yamahachi Dental, Aichi, Japan) using CAD/CAM machine (Imes-icore 250i, Germany).

Groups 3, 4: Vita Enamic and Lava Ultimate specimens ( $4 \mathrm{~mm}$ diameter, $2 \mathrm{~mm}$ height) were designed with CEREC software 4.2 platforms (Sirona Dental GmbH, Salzburg, Austria) and obtained by milling from their respective blocks for CAD/CAM with CEREC MC XL (Sirona Dental Gmbh, Salzburg, Austria).

The materials'names, manufactures, and chemical composition used in this study are listed in Table 1. The specimens were wet ground manually on only one surface using 600-grit silicon carbide paper and then ultrasonically cleaned in distilled water for $5 \mathrm{~min}$.

Table 1. Information regarding the materials used in the study.

\begin{tabular}{|c|c|c|}
\hline Product name & manufacturer & composition \\
\hline Ceramage & Shofu, Kyoto, Japan & $\begin{array}{l}\text { Zirconium Silicate featuring a progressively fine structural filling of more than } 73 \% \\
\text { by weight of micro-fine ceramic particles in an organic polymer matrix }\end{array}$ \\
\hline Yamahachi PMMA & Yamahachi Dental, Aichi, Japan & PMMA \\
\hline Vita Enamic & Vita Zahnfabrik, Bad Säckingen, Germany & $86 w t \%$ feldspar ceramic, $14 w t \%$ polymer \\
\hline Lava Ultimate & 3M ESPE, Seefeld, Germany & $80 \mathrm{wt} \%$ nanoceramic, $20 \mathrm{wt} \%$ resin \\
\hline $\begin{array}{l}\text { RelyX Ultimate } \\
\text { Clicker }\end{array}$ & 3M ESPE, St. Paul, MN, USA & $\begin{array}{l}\text { Base paste: methacrylate monomers, Radiopaque, silanated fillers, initiator, } \\
\text { stabilizers, rheological additives; Catalyst paste: Methacrylate monomers, } \\
\text { radiopaque alkaline fillers, initiators, stabilizers, pigments, rheological additives, } \\
\text { fluorescence dye, dual-cure activator for single bond universal adhesive }\end{array}$ \\
\hline $\begin{array}{l}\text { Single Bond } \\
\text { Universal Adhesive }\end{array}$ & 3M ESPE, St. Paul, MN, USA & $\begin{array}{l}\text { MDP phosphate-monomer dimethacrylate resins, HEMA, vitrbond Copolymer, } \\
\text { filler, ethanol, water, initiators and silane. }\end{array}$ \\
\hline
\end{tabular}

PMMA: Polymethylmethacrylate; MDP: 10-methacryloyloxydecyl dihydrogen phosphate; HEMA: 2-hydroxyetheyl methacrylate.

\subsection{Bonding Procedures}

Before cementation, a small piece of insulating tape with a central hole was placed on the dentin surface. The tape was perforated with a punching machine to create $4 \mathrm{~mm}$ holes. Then, Single Bond Universal Adhesive was applied to the prepared tooth with a microbrush and rubbed in for $20 \mathrm{~s}$. The adhesive was gently air dried for approximately $5 \mathrm{~s}$ to evaporate the solvent, and then light-cured for $10 \mathrm{~s}$, and a thin layer of RelyX ${ }^{\mathrm{TM}}$ Ultimate Clicker ${ }^{\mathrm{TM}}$ adhesive resin cement was applied and placed over the dentin substrates. Subsequently, the specimens were bonded on and excess cement was removed with a microbrush. The adhesive interface was light cured under a load of $10 \mathrm{~N}$ from 4 directions for $40 \mathrm{~s}$. The bonded specimens were stored in distilled water for $24 \mathrm{~h}$ at $(37 \pm 2)^{\circ} \mathrm{C}$ prior to shear bond strength testing.

\subsection{Shear Bond Strength Test}

The shear bond strength was measured using a universal testing machine (MTS, Eden Prairie, USA) at a cross-head 
speed of $1 \mathrm{~mm} / \mathrm{min}$. The force was measured in newtons divided by the cross-sectional area and is reported as megapascals.

\subsection{Statistical Analysis}

SPSS 20 statistical analysis software (IBM, Chicago, USA) were used in this study. As the data was completely numerical, distribution characteristics and homogeneity of variance were checked with the Kolmogorov-Smirnov test and Levene's test, respectively. Descriptive statistics (mean and SD) were computed. Multi-group comparisons of the means were carried out by one-way analysis of variance (ANOVA) test with post hoc contrasts by Student-Newman-Keuls test and Kruska al-Wallis test. All results with $P$-values smaller than 5\% were considered as statistically significant.

\section{Results}

The obtained mean for shear bond strength values and their respective standard deviations of the different polymeric materials are shown in Table 2. Among the tested polymers, the highest shear bond strength values were obtained with Vita Enamic group, which was followed by the Lava Ultimate group. Vita Enamic and Lava Ultimate were not statistically different, and both had significantly higher shear bond strength than the Ceramage and PMMA. The lowest values were obtained for PMMA with significant difference between group PMMA and Ceramage (Figure 1).

Table 2. The shear bond strength (means and standard deviations) of 4 groups.

\begin{tabular}{llll}
\hline Groups & Sample number & Mean (Mpa) & Standard deviation \\
\hline PMMA & 15 & 5.99 & 1.42 \\
Ceramage & 15 & 11.20 & 1.49 \\
Lava Ultimate & 15 & 14.66 & 2.10 \\
Vita Enamic & 15 & 15.43 & 2.69 \\
\hline
\end{tabular}

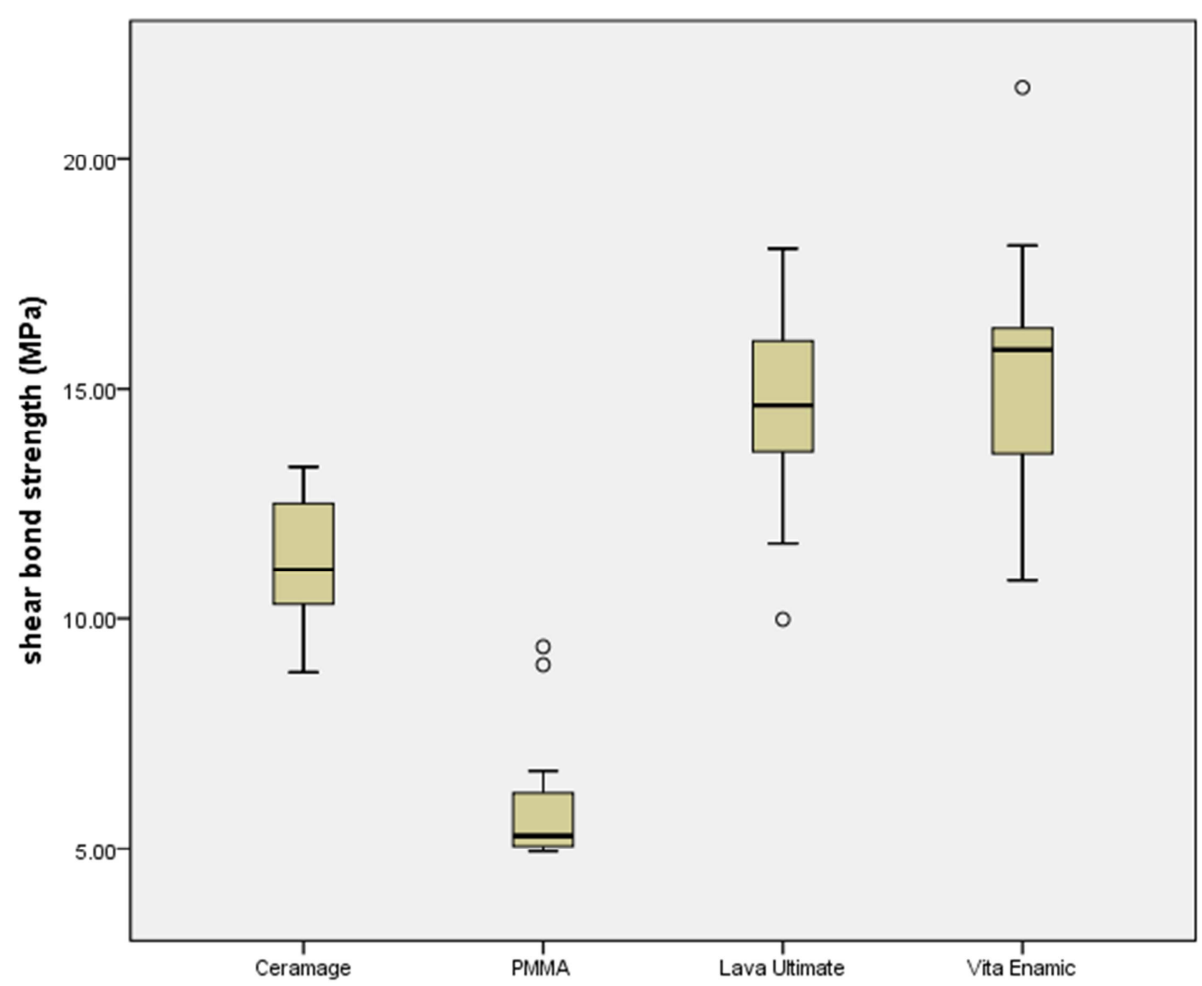

Figure 1. Boxplots for shear bond strength values (MPa; medians, lower and upper quartiles as well as minima and maxima) of the four polymeric materials.

\section{Discussion}

It is well known that different dental restoration materials are priced differently. The consumption ability of patients with tooth defects is widely ranged in China, the biggest developing country. Before different dental restoration materials were used for patients, it is necessary to tell them the merits and demerits of dental restoration materials which are related to factors including repaired dental life at a different prices. Polymeric materials are gaining popularity for hardness, high flexibility, color stability, and with low rigidity, and brittleness [18]. Durable and effective bond strength between the polymeric material and the tooth is fundamental for the clinical long-term survival of a restoration [19]. However, the bond strength of polymeric materials for restorations are not clear. At the same time, some patients need long-term temporaries treatment including changes of vertical dimensions, with considerations of aesthetic and phoneric properties. So it is important for doctors to known shear bond strength value of polymeric dental restoration. The aim of this study was to evaluate the shear bond strength between dentin and four kinds of polymeric materials, which are commonly used in clinic.

In the present study, dentin bond strength of the polymeric materials was determined using the shear bond strength test. Shear bond strength test is defined as a test in which two 
materials are connected by an adhesive agent and loaded in shear until separation occurs [20]. In the shear bond strength test, the shear stress was considered to be more representative of a clinical situation, and the shear bond strength test was considered the most efficient method for reliable results [21].

The shear bond strength of four kinds of polymeric materials which widely used in our clinic were studied. These materials showed different shear bond strength which may depend on their chemical composition. Yamahachi PMMA DISK contains $100 \%$ polymethylmethacrylate without organic or inorganic filler. Ceramage is a kind of light-curing micro-fine resin, containing more than $73 \%$ by weight of micro-fine ceramic particles, which is mainly composed of Zirconium Silicate. Recently, an innovative polymeric $\mathrm{CAD} / \mathrm{CAM}$ material has been introduced. It combines the positive aspects of both ceramics and composites with beneficial properties [22]. Vita Enamic, an innovative polymeric $\mathrm{CAD} / \mathrm{CAM}$ material, is composed of a dominant feldspathic-based ceramic network $(86 \mathrm{wt} \%)$ integrated with an acrylate polymer network $(14 \mathrm{wt} \%)$ with both networks fully penetrating one another [23]. The main components of ceramic network are $\mathrm{SiO}_{2}$ (58 63wt\%), $\mathrm{Al}_{2} \mathrm{O}_{3}(20 \sim 23 \mathrm{wt} \%)$, and $\mathrm{ZrO}_{2}(<1 \mathrm{wt} \%)$. Lava Ultimate, another innovative polymeric CAD/CAM material, which is $80 \mathrm{wt} \%$ nanoceramic particles (silica- and zirconia filler/cluster filler) embedded in a highly cross-linked polymer network (20 wt\%) [24].

In this study, all the specimens were cemented with Single Bond Universal Adhesive, which was advantageous because the Adhesive not only have silane coupling agent, but also have 10-MDP. Application of the adhesive to the Vita Enamic ceramic surfaces can provide a chemical covalent hydrogen bond, which is a major factor for a sufficient bond to ceramics [25]. Besides, Silanes are bifunctional molecules that bond silicon dioxide with $\mathrm{OH}$ groups on the ceramic surface and can increase the bond strength by improving the wettability of surface [26]. They also have a degradable functional group that copolymerizes with the resin's organic matrix [27]. 10-MDP has previously been shown to offer chemical bond-mediating capacity directly with zirconia and thus, to the zirconia clusters/fillers present in Vita Enamic [28, 29]. Therefore, Vita Enamic can have significantly higher bond strength values than other three groups. Compared with Ceramage and PMMA, Lava Ultimate showed higher bond strength values. In this study, although no statistically significant difference found between Lava Ultimate and Vita Enamic, Vita Enamic has shown higher bond strength than Lava Ultimate. This reliable bond between Lava Ultimate and dentin may have been explained through the nanoceramic particles present in Lava Ultimate, and Silanes, 10-MDP monomers present in Adhesive. Lava Ultimate is composed of resin matrix, silica, and zirconia nanomers, getting strong and durable bonding. The bond strength of Ceramage is weaker than that of the Vita Enamic and Lava Ultimate. It is because that, due to a highly uniform and quality ceramic content, the bond strength of Vita Enamic and Lave Ultimate have been increased. The polymerization mode of the Ceramage is greatly influenced by operator, resulting in the presence of flaws during incremental build up, but cannot eliminate during the curing process [30]. PMMA had the lowest shear bond strength values. Reason may be that the PMMA that has high polymethylmethacrylate content and is industrially polymerized at high temperature and pressure, has relatively high density with fewer flaws and pores, which decrease the penetration of adhesive resin cement into the PMMA [31]. In addition, the specific components of PMMA can decrease the possibility of additional chemical bonding, which in turn affect bonding performance. One study showed that most of the functional groups of PMMA did not exist in terms of double bindings, which leads to adhesive problems [32].

Although different polymeric materials showed different shear bond strength value, polymeric material with lower shear bond strength value can be used for long-term tempotizations. Ender and his colleagues have shown that some polymeric materials may be applicable as long-term restorations in some cases [16]. PMMA, the least expensive one in china, showed the lowest shear bond strength value in this study. It can be used for tempotizations more than 6 months [33]. However, It is found that the usage of temporary cements on polymeric materials led to frequent loss of retention through clinical observations. Therefore, doctors should think about repaired dental life which patients wanted before they choose materials.

\section{Conclusions}

Based on the results presented and within the limitations of this study, it can be concluded that four different kinds of polymeric materials showed different shear bond strength. The bond strength of polymeric materials is material dependent. Vita Enamic, Lava Ultimate and Ceramage revealed higher shear bond strength than PMMA. Despite the high cost of treatment, Vita Enamic, Lava Ultimate and Ceramage restorations should be advised if the retained tooth is expected to maintain functionality over the long time. PMMA, the least expensive one in china, showed the lowest shear bond strength value in this study. It can be used for long-term tempotizations more than 6 months. Since the shear bond strength differed, it is evident that appropriate polymeric materials selection should be based on considerations of specific clinical situations and economic levels. So doctors can use them for patients with different intent.

\section{References}

[1] Kassem AS, Atta O, El-Mowafy O. Fatigue resistance and microleakage of $\mathrm{CAD} / \mathrm{CAM}$ ceramic and composite molar crowns. J Prosthodont 2012; 21 (1): 28-32.

[2] Barizon KT, Bergeron C, Vargas MA, Qian F, Cobb DS, Gratton DG, Geraldeli S. Ceramic materials for porcelain veneers: Part II. Effect of material, shade, and thickness on translucency. J Prosthet Dent 2014; 112 (4): 864-870.

[3] Ruse ND, Sadoun MJ. Resin-composite Blocks for Dental CAD/CAM Applications. J Dent Res 2014; 93 (12): 1232 1234. 
[4] Mörmann WH, Stawarczyk B, Ender A, Sener B, Attin T, Mehl A. Wear characteristics of current aesthetic dental restorative CAD/CAM materials: two-body wear, gloss retention, roughness and Martens hardness. J Mech Behav Biomed Mater 2013; 20: 113-125.

[5] Xing W, Jiang T, Ma X, Liang S, Wang Z, Sa Y, Wang Y. Evaluation of the esthetic effect of resin cements and try-in pastes on ceromer veneers. J Dent 2010; 38 Suppl 2: e87-94.

[6] Dukic W, Dukic OL, Milardovic S, Delija B. Clinical evaluation of indirect composite restorations at baseline and 36 months after placement. Oper Dent 2010; 35 (2): 156-164.

[7] Ferracane JL. Resin composite-State of the art. Dent Mater 2011; 27 (1): 29-38.

[8] da Rosa Rodolpho PA, Cenci MS, Donassollo TA, Loguércio $\mathrm{AD}$, Demarco FF. A clinical evaluation of posterior composite restorations: 17-year findings. J Dent 2006; 34 (7): 427-435.

[9] Grivas E, Roudsari RV, Satterthwaite JD. Composite inlays: a systematic review. Eur J Prosthodont Restor Dent 2014; 22 (3): 117-124.

[10] Carvalho AO, Bruzi G, Giannini M, Magne P. Fatigue resistance of $\mathrm{CAD} / \mathrm{CAM}$ complete crowns with a simplified cementation process. J Prosthet Dent 2014; 111 (4): 310-317.

[11] Stawarczyk B, Özcan M, Trottmann A, Schmutz F, Roos M, Hämmerle C. Two-body wear rate of CAD/CAM resin blocks and their enamel antagonists. J Prosthet Dent 2013; 109 (5): 325-332.

[12] Krämer N, Kunzelmann KH, Taschner M, Mehl A, Garcia-Godoy F, Frankenberger R. Antagonist enamel wears more than ceramic inlays. J Dent Res 2006; 85 (12): 1097-1100.

[13] Magne P, Perakis N, Belser UC, Krejci I. Stress distribution of inlay-anchored adhesive fixed partial dentures: a finite element analysis of the influence of restorative materials and abutment preparation design. J Prosthet Dent 2002; 87 (5): 516-527.

[14] Rocca GT, Bonnafous F, Rizcalla N, Krejci I. A technique to improve the esthetic aspects of CAD/CAM composite resin restorations. J Prosthet Dent 2010; 104 (4): 273-275.

[15] Lin CL, Chang YH, Liu PR. Multi-factorial analysis of a cusp-replacing adhesive premolar restoration: a finite element study. J Dent 2008; 36 (3): 194-203.

[16] Ender A, Bienz S, Mörmann W, Mehl A, Attin T, Stawarczyk B. Marginal adaptation, fracture load and macroscopic failure mode of adhesively luted PMMA-based CAD/CAM inlays. Dent Mater 2016; 32 (2): e22-29.

[17] Poggio C, Pigozzo M, Ceci M, Scribante A, Beltrami R, Chiesa M. Influence of different luting protocols on shear bond strength of computer aided design/computer aided manufacturing resin nanoceramic material to dentin. Dent Res J 2016; 13 (2): 91-97.

[18] Elsaka SE. Bond strength of novel CAD/CAM restorative materials to self-adhesive resin cement: the effect of surface treatments. J Adhes Dent 2014; 16 (6): 531-540.

[19] Bähr N, Keul C, Edelhoff D, Eichberger M, Roos M, Gernet W, Stawarczyk B. Effect of different adhesives combined with two resin composite cements on shear bond strength to polymeric CAD/CAM materials. Dent Mater J 2013; 32 (3): 492-501.
[20] Al-Dohan HM, Yaman P, Dennison JB, Razzoog ME, Lang BR Shear strength of core-veneer interface in bi-layered ceramics. J Prosthet Dent 2004; 91 (4): 349-355.

[21] Cardoso PE, Braga RR, Carrilho MR. Evaluation of micro-tensile, shear and tensile tests determining the bond strength of three adhesive systems. Dent Mater 1998; 14 (6): 394-398.

[22] Goujat A, Abouelleil H, Colon P, Jeannin C, Pradelle N, Seux $\mathrm{D}$, Grosgogeat B. Mechanical properties and internal fit of 4 CAD-CAM block materials. J Prosthet Dent 2018; 119 (3): 384-389.

[23] Vita Enamic. Vita Enamic Concept Brochure. Available at https://www.vita-zahnfabrik.com/datei.php?src=portal/sap/dat eien/c/cc0/cc0c01/Konzeptbroschuere/VITA_10024E_ENAM IC_PS_EN_V02.Pdf.

[24] Lava Ultimate CAD/CAM Restorative. Lava Ultimate Brochure. Available at http://multimedia.3m. $\mathrm{com} / \mathrm{mws} /$ mediawebserver?Mwsld=66666UgxGCuNyXTt08T _oxT6EVtQEcuZgVs6E666666-\&fn=Lava_Ultimate_F_UK.p $\overline{\mathrm{d} f}$.

[25] www.3mespe.com/Single bond universal [Online].

[26] Soares CJ, Giannini M, Oliveira MT, Paulillo LA, Martins LR. Effect of surface treatments of laboratory-fabricated composites on the microtensile bond strength to a luting resin cement. J Appl Oral Sci 2014; 12 (1): 45-50.

[27] Söderholm KJ, Shang SW. Molecular orientation of silane at the surface of colloidal silica. J Dent Res 1993; 72 (6): 1050-1054.

[28] Flury S, Schmidt SZ, Peutzfeldt A, Lussi A. Dentin bond strength of two resin-ceramic computer-aided design/computer-aided manufacturing $(\mathrm{CAD} / \mathrm{CAM})$ materials and five cements after six months storage. Dent Mater J 2016; 35 (5): $728-735$.

[29] Cekic-Nagas I, Ergun G, Egilmez F, Vallittu PK, Lassila LV. Micro-shear bond strength of different resin cements to ceramic/glass-polymer $\mathrm{CAD} / \mathrm{CAM}$ block materials. J Prosthodont Res 2016; 60 (4): 265-273.

[30] Leinfelder KF. New developments in resin restorative systems. J Am Dent Assoc 1997; 128 (5): 573-581.

[31] Hanabusa M, Mine A, Kuboki T, Momoi Y, Van Ende A, Van Meerbeek B, De Munck J. Bonding effectiveness of a new 'multi-mode' adhesive to enamel and dentine. J Dent 2012; 40 (6): 475-484.

[32] Liebermann A, Keul C, Bähr N, Edelhoff D, Eichberger M, Roos M, Stawarczyk B. Impact of plasma treatment of PMMA-based CAD/CAM blanks on surface properties as well as on adhesion to self-adhesive resin composite cements. Dent Mater 2013; 29 (9): 935-944.

[33] Huetting F, Prutscher A, Goldammer C, Kreutzer CA, Weber H. First clinical experiences with $\mathrm{CAD} / \mathrm{CAM}$-fabricated PMMA-based fixed dental prostheses as long-term temporaries. Clin Oral Investing 2016; 20 (1): 161-168. 